\title{
Interaction of Two Complementary Fragments of the Bovine Spinal Cord Myelin Basic Protein with Phospholipid Bilayers. An ESR Spin-Label Study ${ }^{\dagger}$
}

\author{
M. B. Sankaram, ${ }^{\ddagger}$ P. J. Brophy, ${ }^{\S}$ and D. Marsh ${ }^{*, \ddagger}$ \\ Max-Planck-Institut für biophysikalische Chemie, Abteilung Spektroskopie, Postfach 2841, D-3400 Göttingen. \\ Federal Republic of Germany, and Department of Biological Science, University of Stirling, Stirling FK 4 LA, \\ United Kingdom \\ Received December 9, 1988; Revised Manuscript Received July 24, 1989
}

\begin{abstract}
The myelin basic protein (MBP) from bovine spinal cord was cleaved at the single tryptophan residue to produce an $\mathrm{N}$-terminal fragment (F1) of molecular weight $12.6 \mathrm{~K}$ and a $\mathrm{C}$-terminal fragment (F2) of molecular weight $5.8 \mathrm{~K}$. The interactions of the two fragments with bilayers of the acidic lipid dimyristoylphosphatidylglycerol (DMPG) were compared with those of the intact protein, by using both chemical binding assays and spin-label electron spin resonance spectroscopy. The saturation binding stoichiometries of the two fragments were found to sum to that of the MBP, having values of 11,24 , and $36 \mathrm{~mol}$ of DMPG/mol of protein for F2, F1, and the MBP, respectively. The strength of binding was found to increase in the order $F 2<F 1<M B P$, which follows that of the net charges on the different fragments. The ionic strength dependence of the protein binding indicated that the interaction is primarily of electrostatic origin. The efficiency of displacement of the proteins by salt was in the order F2 > F1 > MBP, which correlates with both the strength of binding and the net charge on the different protein fragments. Nitroxide derivatives of phosphatidylglycerol (PG) labeled on the $s n-2$ chain were used to probe the protein-induced changes in the acyl chain dynamics. Both the fragments and the MBP decreased the lipid chain mobility as recorded by the C- 5 atom and C- 12 atom position nitroxide-PG spin-labels, in a manner which followed the protein binding curves. At saturation binding, the reduction in mobility recorded by the $\mathrm{C}-5$ atom label was in the order MBP > F1 > F2. An additional population of lipids, whose chain motion was restricted relative to that of the bulk population of fluid lipids, was resolved in the case of the F1 fragment and the MBP, when using the $\mathrm{C}-12$ atom position labeled PG. Approximately nine DMPG molecules per F1 fragment were found in this motionally restricted population, which was assigned to lipids in direct contact with partially penetrant sections of the protein, as opposed to a $18: 1 \mathrm{~mol} / \mathrm{mol}$ lipid/protein stoichiometry found for the restricted component with the intact MBP. These results suggest that the principal sites of hydrophobic interaction of the protein with lipid bilayers are at least partly located in the $12.6-\mathrm{kDa}$ fragment. The tryptophan residue at position 116 appears also to be important for the structural and functional properties of the intact bovine myelin basic protein.
\end{abstract}

$T_{\text {he }}$ plays an important role in the structural organization of the myelin sheath [see Boggs and Moscarello (1978a,b) and Boggs et al. (1982)]. Induction and maintenance of the multilamellar structure of myelin are thought to be brought about by a bridging of apposing cytoplasmic surfaces. A hairpin structure of the protein caused by a tri-proline sequence approximately in the middle of the molecule is considered to cause interlamellar interactions (Eylar et al., 1971).

Insights into the nature of the association have been gained by physicochemical studies on the interaction of MBP with lipid bilayers. Thus, this protein has been shown to bind strongly to the headgroups of acidic lipids (Palmer \& Dawson, 1969; Sankaram et al., 1989). Both electrostatic and hydrophobic components of the interaction have been detected by using differential scanning calorimetry (Papahadjopoulos et al., 1975; Boggs et al., 1978a). Binding to the negatively charged lipid headgroups is obligatory for an effective association of the protein with lipid bilayers, as has been demonstrated by spin-label ESR spectroscopy and chemical binding studies (Sankaram et al., 1989). The protein has been found

\footnotetext{
${ }^{\dagger}$ M.B.S. is the recipient of a fellowship from the Alexander von Humboldt Stiftung.

${ }^{\ddagger}$ Max-Planck-Institut für biophysikalische Chemie.

${ }^{8}$ University of Stirling.
}

to exhibit a diverse conformational variability both in solution (Eylar \& Thompson, 1969; Chao \& Einstein, 1970; Palmer \& Dawson, 1969; Liebes et al., 1975; Stone et al., 1985; Mendz et al., 1984) and when bound to lipids (Surewicz et al., 1987; Keniry \& Smith, 1979).

In an attempt to define specific regions of the protein that contribute to its native conformation in the myelin sheath, the interactions between lipids and several fragments of the protein produced by cleaving with cathepsin (Keniry \& Smith, 1979) and BNPS-skatole (London et al., 1973; Jones \& Rumsby, 1977; Boggs et al., 1981) have been studied. BNPS-skatole cleaves the bovine protein at the single tryptophan residue, producing a large $\mathrm{N}$-terminal fragment (residues 1-116), F1, of molecular weight $12.6 \mathrm{~K}$ and a relatively small C-terminal fragment (residues 117-170), F2, of molecular weight 5.8K (Martenson et al., 1975). Biphasic solvent extraction experiments on these fragments have shown that the intact protein and the fragment $\mathrm{F} 2$ formed chloroform-soluble complexes

\footnotetext{
Abbreviations: MBP, bovine spinal cord myelin basic protein; ESR, electron spin resonance; $\mathrm{F} 1$, the $\mathrm{N}$-terminal $12.6-\mathrm{kDa}$ fragment of myelin basic protein; $\mathrm{F} 2$, the $\mathrm{C}$-terminal $5.8-\mathrm{kDa}$ fragment of myelin basic protein; EDTA, ethylenediaminetetraacetic acid; Tris, tris(hydroxymethyl)aminomethane; $n$-PGSL, 1 -acyl-2-[n-(4,4-dimethyloxazolidine$N$-oxyl)stearoyl]-sn-glycero-3-phosphoglycerol; DMPG, 1,2-dimyristoyl-sn-glycero-3-phosphoglycerol; BNPS-skatole, 2-[(2-nitrophenyl)sulfenyl]-3-methyl-3-bromoindolenine.
} 
with acidic lipids such as phosphatidylserine, phosphatidylinositol, and sulfatide, while the fragment Fl did not (Jones \& Rumsby, 1977). In contrast to these observations, differential scanning calorimetric studies of the complexes of human MBP, and the fragments F1 and F2, with dipalmitoylphosphatidylglycerol and dimyristoylphosphatidic acid have shown that the MBP and the F1 fragment interacted hydrophobically in a similar way, while the F2 fragment showed quantitatively less effect (Boggs et al., 1981). A study of the interaction of the $\mathrm{N}$ - and $\mathrm{C}$-terminal fragments with lipid monolayers led to the conclusion that the $F 1$ fragment interacted hydrophobically with the lipid to a greater extent than did the F2 fragment (London et al., 1973). Two catheptic peptides (residues 1-89 and 90-170) have been found to bind to lipid bilayers to a similar extent, on a $w / w$ basis, as did the intact bovine protein (Keniry \& Smith, 1979). However, the carboxy-terminal peptide showed no increase in helical content on binding, and only minor changes were observed with the amino-terminal peptide, as opposed to the response of the intact native protein which underwent a conformational transition to a more folded state. Thrombic peptides of rabbit MBP (residues 1-95 and 96-168) have been shown to differ in their conformational adaptability in aqueous trifluoroethanol solutions (Stone et al., 1985).

In addition to its role in the structural stabilization of myelin, the MBP induces an autoimmune disease, experimental allergic encephalomyelitis, in animals. Tests of encephalitogenic activity in Lewis rats have shown that the F1 and F2 fragments of bovine MBP were much less active than the uncleaved protein (Martenson et al., 1975). Whereas the major encephalitogenic site of guinea pig MBP is confined to the region comprising residues 68-88 (Chou et al., 1977), the fragment 43-88 from bovine MBP is inactive. Although it appears fairly certain that scission of bovine MBP at the tryptophan residue has a marked effect on protein structure and activity, conflicting conclusions have been drawn regarding the location of the principal sites for hydrophobic interaction (London et al., 1973; Jones \& Rumsby, 1977; Boggs et al., 1981).

Spin-label ESR spectroscopy provides a potential method to identify and quantitate hydrophobic lipid-protein associations [see, e.g., Marsh (1985) and Görrissen et al. (1986)]. Studies on the binding of the intact MBP to dimyristoylphosphatidylglycerol (DMPG) bilayers have demonstrated the existence of a population of lipids whose chain motions are restricted by direct interaction with the protein (Sankaram et al., 1989). We report here our results on a comparative study of the effects of the native MBP and the BNPS-skatole-cleaved fragments on DMPG bilayers. Salt titration has been used to estimate the relative strengths of the electrostatic interactions, and ESR difference spectroscopy to locate the putative membrane-penetrant sections of the protein. In addition, binding curves have been constructed to assess the saturation binding of the different fragments.

\section{Materials AND MethodS}

Materials. DMPG was prepared from dimyristoylphosphatidylcholine (Fluka, Buchs, Switzerland) by phospholipase D (Boehringer-Mannheim, West Germany) catalyzed transphosphatidylation (Comfurius \& Zwaal, 1977). Positional isomers, $n$-PGSL, of spin-labeled phosphatidylglycerol were synthesized as described in Marsh and Watts (1982). Egg lysophosphatidylcholine (Lipid Products, S. Nutfield, U.K.) was used for the synthesis. Therefore, the composition of the $s n-1$ chain is mostly palmitoyl. The spinlabels were stored at $-20^{\circ} \mathrm{C}$ in $\mathrm{CH}_{2} \mathrm{Cl}_{2}$ at a concentration of $1 \mathrm{mg} / \mathrm{mL}$.
Protein Isolation. Bovine spinal cord was obtained fresh from the slaughterhouse and frozen immediately in liquid $\mathrm{N}_{2}$. Myelin basic protein was extracted and purified as described by Deibler et al. (1972). The protein was further purified by chromatography on a Sephadex G-75 Superfine column $(3 \times$ $90 \mathrm{~cm})$ equilibrated with $10 \mathrm{mM} \mathrm{HCl}$. The protein $\left(M_{\mathrm{r}}\right.$ 18400 ) was pure as judged by polyacrylamide gel electrophoresis in sodium dodecyl sulfate (Laemmli, 1970). Protein concentrations were measured spectrophotometrically by using a value for the extinction of $E_{1 \%}^{276.4 \mathrm{~nm}}=5.64$ (Liebes et al., 1975).

Preparation of Protein Fragments. The basic protein was cleaved at the single tryptophan residue with BNPS-skatole by the method of Martenson et al. (1975). The two peptide fragments were isolated, and the methionine residues were regenerated as described by Jones and Rumsby (1977). Final chromatography of each peptide on Sephadex G-75 Superfine yielded one symmetrical peak of protein which stained as a single band on analysis by SDS-PAGE (Laemmli, 1970).

Sample Preparation. Appropriate amounts of DMPG (1 $\mathrm{mg}$ ) and $n$-PGSL ( $1 \mathrm{~mol} \%$ ) were dissolved in $\mathrm{CH}_{2} \mathrm{Cl}_{2}$. A thin film was formed by evaporating the solvent under a stream of $\mathrm{N}_{2}$ gas and drying under vacuum in a desiccator for at least $3 \mathrm{~h}$. The thin films were hydrated by adding $50 \mu \mathrm{L}$ of buffer (10 mM Tris, $10 \mathrm{mM} \mathrm{NaCl}$, and $0.1 \mathrm{mM}$ EDTA, $\mathrm{pH} \mathrm{8.0)}$ and gentle mixing. The required amount of protein solution was added to the lipid dispersion thus obtained, in the same buffer. The lipid-protein complexes precipitated instantaneously. Alternatively, the dry lipid films were hydrated directly with the protein solution. Complexation of the protein with DMPG was complete, irrespective of the method of reconstitution employed. Recombinants at high lipid:protein ratios were pelleted at $30000 \mathrm{~g}$ in a $65 \mathrm{Ti}$ rotor, whereas recombinants with a saturating amount of protein were centrifuged on a bench centrifuge at $3000 \mathrm{rpm}$. The recombinant pellet was washed several times in the above buffer and then transferred to a 100- $\mu \mathrm{L}$ capillary for ESR spectroscopy. No protein was detected in the supernatant from the washes. Sucrose density gradient centrifugation showed that the lipid-protein complexes sedimented as a single band, and therefore were homogeneous with respect to lipid:protein ratio. The samples were dissolved, after the ESR experiments, in $1 \mathrm{~N} \mathrm{NaOH}$ solution to estimate the lipid and protein contents according to Eibl and Lands (1969) and Lowry et al. (1951), respectively. Samples for the salt dependence experiments were prepared as described above, in $\mathrm{pH} 8.0$ buffers containing $10 \mathrm{mM}$ Tris, $0.1 \mathrm{mM}$ EDTA, and varying concentrations of $\mathrm{NaCl}$ in the range $0-2 \mathrm{M}$. In all cases, an excess of protein solution ( 2.5 $\mathrm{mg}$ of protein $/ \mathrm{mg}$ of lipid) was added to ensure saturation binding.

ESR Spectroscopy. ESR spectra were recorded on a Varian E-12 Century Line 9-GHz spectrometer equipped with a nitrogen gas flow temperature regulation system. The sealed capillaries (1-mm diameter) were placed in a quartz tube containing silicone oil for thermal stability. A PDP $11 / 10$ dedicated computer system with interactive graphics was used for digital data collection and for data processing. Spectral subtractions were performed as previously described (Marsh, 1982; Görrissen et al., 1986). Effective order parameters, $S_{\text {eff }}$, were calculated as described previously (Sankaram et al., 1989). Detailed ESR line-shape simulations have shown that the spectra of lipid spin-labels contain important contributions from slow molecular motions (Lange et al., 1985). Therefore, the effective order parameters calculated by using motional narrowing theory can only be considered as apparent values, 


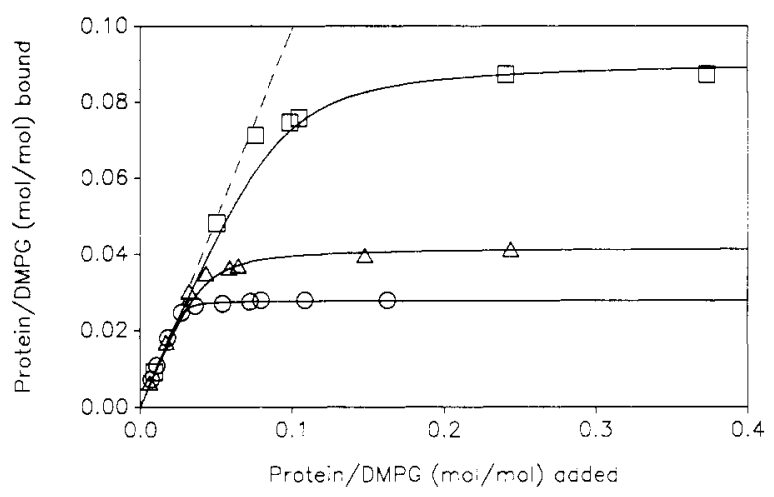

FIGURE 1: Dependence of the binding (expressed as mole ratio) of the MBP (O), the F1 fragment ( $\Delta)$, and the F2 fragment ( $\square)$, to DMPG dispersions, on the total protein to lipid mole ratio. Buffer: $10 \mathrm{mM}$ Tris, $10 \mathrm{mM} \mathrm{NaCl}$, and $0.1 \mathrm{mM}$ EDTA, pH 8.0. The dashed line indicates the theoretical dependence when all of the added protein is bound. The solid lines represent simple mass action binding curves calculated from the effective binding constants deduced from the data points between the linear and saturation regions, as explained under Discussion.

but are nonetheless useful for investigating the comparative effects of the different protein fragments.

\section{RESULTS}

Protein Binding. The MBP and its fragments F1 and F2 bind strongly to acidic lipid bilayers. The kinetics of binding are rapid (Young et al., 1982), as seen by an instantaneous precipitation of the lipid-protein complexes upon addition of the protein. Binding curves for the MBP, and the fragments $F 1$ and F2, are given in Figure 1. In all the three cases, an almost quantitative binding of the added protein is observed below saturation concentrations. As saturation is approached, the strength of binding decreases in the order MBP $>\mathrm{F} 1>$ F2. At saturation binding, $1 \mathrm{~mol}$ of protein is bound per 36 , 24 , and $11 \mathrm{~mol}$ of DMPG for the MBP, fragment F1, and fragment $F 2$, respectively.

ESR Spectroscopy. The ESR spectra of the 5-PGSL phosphatidylglycerol spin-label in pure DMPG dispersions are compared with those in the lipid-protein fragment complexes at saturation binding, in the upper part of Figure 2. The spectra of pure DMPG dispersions in the fluid phase at $40^{\circ} \mathrm{C}$ correspond to an axially symmetric, partially motionally averaged anisotropic system. Binding of the protein fragments has the effect of decreasing the degree of motional averaging, which is reflected as an increase in the outer hyperfine splitting, $2 A_{\max }$, and a decrease in the inner hyperfine splitting, $2 A_{\min }$. The extent of the protein-induced increase in the hyperfine anisotropy, $A_{\max }-A_{\min }$, and the apparent order parameter, $S_{\text {eff }}$, decreased in the order MBP $>$ F1 $>$ F2. The dependence of the outer hyperfine splitting on protein:lipid ratio is shown in Figure 3. In the region where the binding curves for the various fragments are superimposable (Figure 1), the values of $A_{\max }$ are also very similar. However, the limiting values of $A_{\max }$ are different for the three proteins, as mentioned above.

The effects of binding of the MBP, and of the F1 and F2 fragments, on the ESR spectra of the 12-PGSL spin-label in DMPG bilayers are shown in the lower part of Figure 2. Pure DMPG bilayers labeled with 12-PGSL give narrow motionally averaged spectral line shapes in the fluid phase at $40^{\circ} \mathrm{C}$. Protein complexation has a general effect of broadening and increasing the anisotropy of these spectra. In addition, the spectra of the MBP and F1 fragment complexes of DMPG are two-component in nature. This is most clearly seen in the spectra recorded at $30^{\circ} \mathrm{C}$ which are given in Figure $4 \mathrm{~A}, \mathrm{~B}$. One of the components is broader and is resolved in the outer

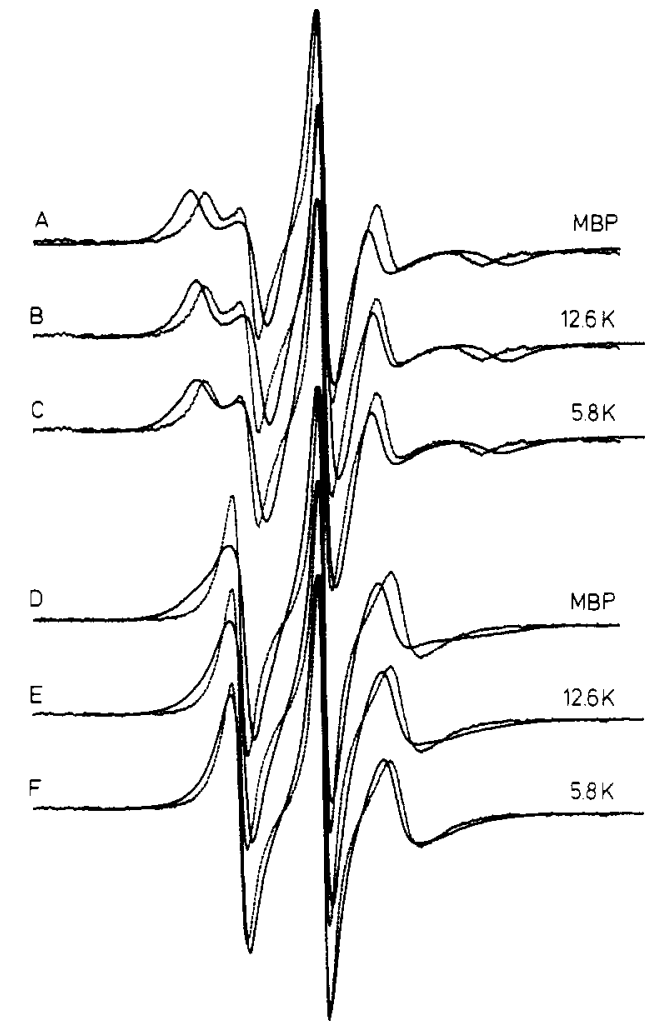

FIGURE 2: ESR spectra of the 5-PGSL (A-C) and the 12-PGSL (D-F) phosphatidylglycerol spin-labels in DMPG-protein fragment complexes. Dotted lines correspond to pure DMPG bilayers, and solid lines correspond to complexes with MBP (A and D), F1 fragment ( $B$ and $E$ ), and $F 2$ fragment ( $C$ and $F$ ). The lipid:protein mole ratios of the complexes were $36: 1$ (MBP), 24:1 (F1 fragment), and 11:1 (F2 fragment), respectively. Spectra recorded at $40^{\circ} \mathrm{C}$; scan width $=100 \mathrm{G}$.

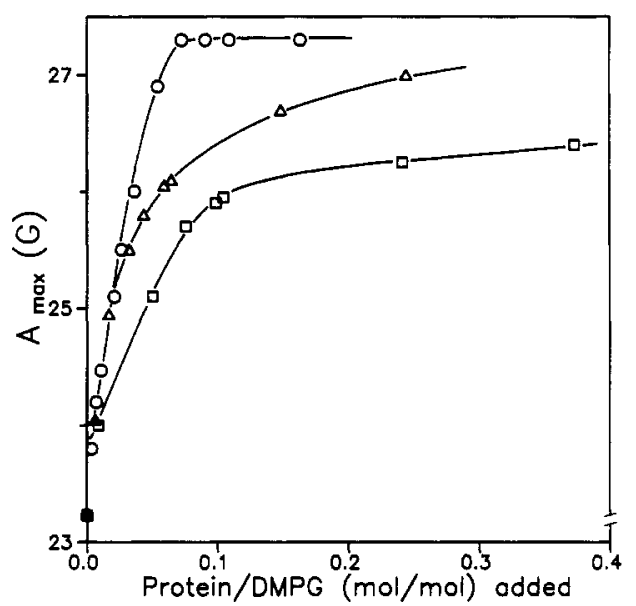

FIGURE 3: Dependence of the outer hyperfine splitting, $2 A_{\max }$, for the 5-PGSL phosphatidylglycerol spin-label in DMPG dispersions at $40^{\circ} \mathrm{C}$ on added protein, for complexes with the MBP (O), F1 fragment $(\Delta)$, and F2 fragment $(\square)$.

wings of the spectrum. By analogy with the results with integral proteins, this component is assigned to lipids whose chain motion is restricted by direct interaction of the chains with the protein. The other component, which dominates the central part of the spectrum and is similar to that found in the pure lipid dispersions, represents the fluid lipids in the bilayer. The relative proportions of these two spectral components were determined by spectral subtractions as shown in Figure 4. The C-terminal fragment, F2, did not exhibit such resolved two-component spectra. Other possible interpretations of the two-component nature of the 12-PGSL spectra have 


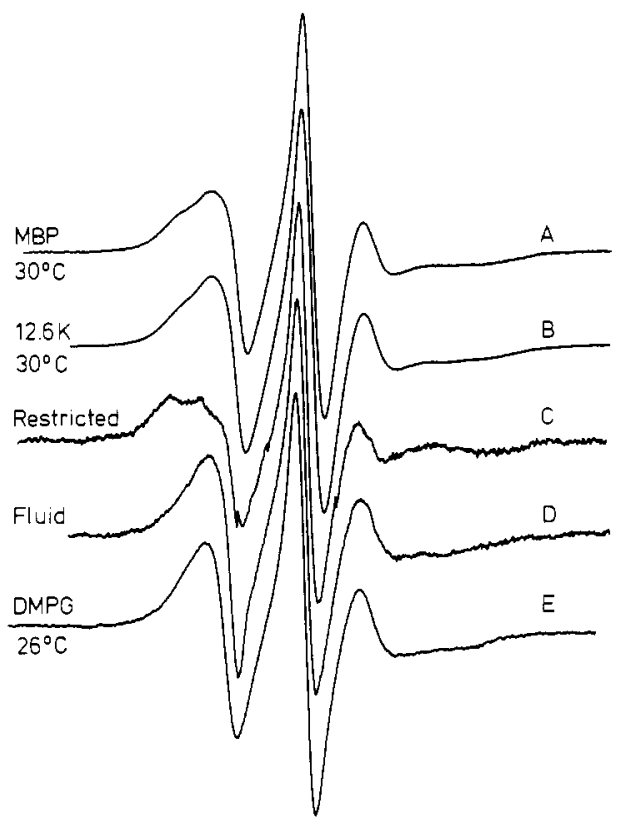

FIGURE 4: Spectral intersubtraction with the 12-PGSL spin-label. Experimental ESR spectra at $30^{\circ} \mathrm{C}$ of DMPG-protein recombinants with (A) the MBP and (B) the F1 fragment. Spectrum C is the difference spectrum obtained by subtracting spectrum B from spectrum A. Spectrum $D$ is the difference spectrum obtained by subtracting spectrum A from spectrum B. Spectrum E is the experimental ESR spectrum of a pure DMPG multilamellar dispersion, recorded at 26 ${ }^{\circ} \mathrm{C}$. Total scan width $=100 \mathrm{G}$.

been considered previously in connection with the MBP (Sankaram et al., 1989). No motionally restricted component was observed with 16-PGSL for any of the fragments. That with 14-PGSL yielded similar results with MBP as for 12PGSL (Sankaram et al., 1989).

Spectral Subtractions. Digital spectral subtractions were performed on normalized ESR spectra of the 12-PGSL label in DMPG-MBP (Figure 4A) and DMPG-F1 (Figure 4B) complexes. The motionally restricted components seen in the outer wings of these two spectra are similar in line shape but differ in amount. Hence, subtracting spectrum 4B from spectrum 4A leads to a difference spectrum representing the motionally restricted lipid component (Figure 4C). The difference spectrum obtained by subtracting spectrum 4A from spectrum $4 B$ represents the spectrum of the fluid lipid population in the two recombinants and is given by Figure 4D. This spectrum is broader than the experimental spectrum of the 12-PGSL-labeled pure DMPG bilayers at $30^{\circ} \mathrm{C}$ and approximates the pure lipid spectrum obtained at $26^{\circ} \mathrm{C}$ (Figure $4 \mathrm{E})$. This broadening is a reflection of the increased spectral anisotropy which was observed for the 5-PGSL label. The internal consistency of the intersubtraction method was monitored by verifying that similar difference spectra were obtained by subtracting fluid lipid spectra from the two-component spectra as those obtained by intersubtraction. The relative amounts of the fluid and motionally restricted lipids in both the DMPG-MBP and DMPG-F1 complexes were determined from the two linear equations:

$$
\begin{gathered}
S^{\mathrm{MBP}}=X_{\mathrm{F}}^{\mathrm{MBP}} S^{\mathrm{F}}+\left(1-X_{\mathrm{F}}^{\mathrm{MBP}}\right) S^{\mathrm{MR}} \\
S^{\mathrm{F} 1}=X_{\mathrm{F}}{ }^{\mathrm{F} 1} S^{\mathrm{F}}+\left(1-X_{\mathrm{F}}{ }^{\mathrm{F} 1}\right) S^{\mathrm{MR}}
\end{gathered}
$$

where $S^{\mathrm{MBP}}$ and $S^{\mathrm{Fl}}$ are the two-component spectra of the DMPG-MBP (Figure 4A) and DMPG-Fl (Figure 4B) complexes, respectively. $S^{\mathrm{F}}$ and $S^{\mathrm{MR}}$ represent ESR spectra corresponding to the fluid (Figure 4D) and the motionally restricted (Figure $4 \mathrm{C}$ ) population of lipids, respectively. $X_{\mathrm{F}}{ }^{\mathrm{MBP}}$

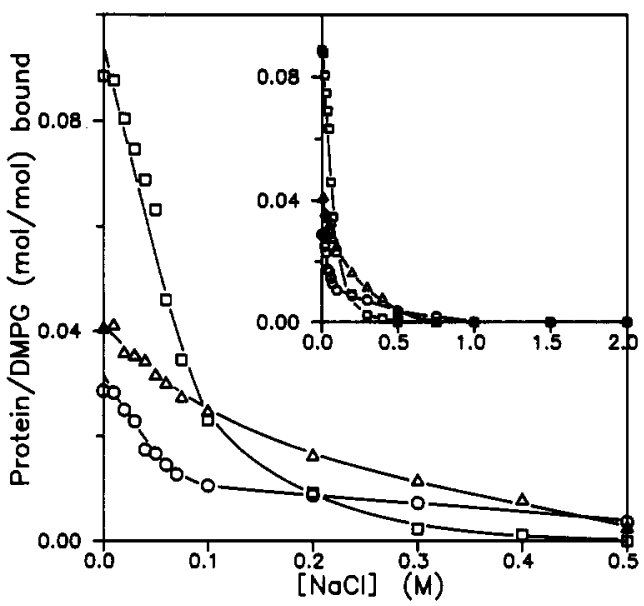

FIGURE 5: Dependence of saturation binding expressed as the mole ratio, protein/DMPG $(\mathrm{mol} / \mathrm{mol})$, on $\mathrm{NaCl}$ concentration in the range $0-0.5 \mathrm{M}$ for the MBP (O), F1 fragment $(\Delta)$, and F2 fragment ( $\square$ ). The inset shows the data for the full $\mathrm{NaCl}$ concentration range studied $(0-2 \mathrm{M})$

and $X_{\mathrm{F}}{ }^{\mathrm{F}}$ are the fractions of fluid lipids for the DMPG-MBP and DMPG-F1 complexes, respectively. The subtraction end point given by the difference spectrum of Figure $4 \mathrm{C}$ therefore corresponds to subtraction of a fraction $X_{\mathrm{F}}^{\mathrm{MBP}} / X_{\mathrm{F}}^{\mathrm{F} 1}$ of the total integrated intensity, and for the complementary subtraction, this factor is $\left(1-X_{\mathrm{F}}^{\mathrm{Fl}}\right) /\left(1-X_{\mathrm{F}}^{\mathrm{MBP}}\right)$.

The results of intersubtractions showed that the motionally restricted component has lipid/protein stoichiometries of 18:1 and 9:1 $\mathrm{mol} / \mathrm{mol}$ for the DMPG-MBP and DMPG-F1 fragment complexes, respectively. The validity of the analysis of the 12-PGSL spectra in terms of two components is further demonstrated by the fact that the lipid/protein stoichiometry for the intact MBP determined by different subtraction strategies $(18: 1 \mathrm{~mol} / \mathrm{mol})$ agrees well with this result (Sankaram et al., 1989). Similar results were obtained with intersubtractions between spectra recorded at $35^{\circ} \mathrm{C}$. It was not possible to obtain satisfactory intersubtractions with spectra recorded at $40^{\circ} \mathrm{C}$ because the temperature dependence of the splitting of the motionally restricted component is considerably greater than that found with integral membrane proteins. For MBP itself, it was, however, possible to obtain satisfactory end points in direct subtractions with spectra recorded at $40^{\circ} \mathrm{C}$ (Sankaram et al., 1989).

Ionic Strength Dependence. Addition of salt has a strong effect on both protein binding and the ESR spectral parameters. The dependence of the binding of the different fragments on ionic strength is shown in Figure 5. Protein binding is abolished at $\mathrm{NaCl}$ concentrations above 1.5 M. Highly significant is the fact that the salt dependence of protein binding is different for the three proteins. The effectiveness of $\mathrm{NaCl}$ in depleting the membrane of protein decreases in the order F2 $>$ F1 $>$ MBP. Only for salt concentrations $<0.1$ $\mathrm{M} \mathrm{NaCl}$ is $\mathrm{F} 1$ displaced less steeply than is MBP. Total displacement of the protein occurs in the concentration ranges $0.3-0.5,0.5-0.75$, and $0.75-1.0 \mathrm{M} \mathrm{NaCl}$ for the $\mathrm{F} 2$ fragment, the $\mathrm{F} 1$ fragment, and the MBP, respectively.

The outer hyperfine splittings $\left(A_{\max }\right)$ from the ESR spectra of 5-PGSL at $40^{\circ} \mathrm{C}$ in the DMPG-MBP, DMPG-F1, and DMPG-F2 complexes decrease as a function of added $\mathrm{NaCl}$ concentration, in a manner which parallels the results for the protein binding given in Figure 5 (data not shown). The difference in the salt-dependent effects for the three protein fragments is also evident from the spin-label ESR spectra. The steepness of decrease in the hyperfine anisotropy is in the order F2 $>$ F1 $>$ MBP. The outer hyperfine splitting is reduced 
to that for the lipid alone at $\mathrm{NaCl}$ concentrations of approximately $0.3,0.5$, and $1.0 \mathrm{M}$ for the $\mathrm{F} 2$ fragment, the $\mathrm{F} 1$ fragment, and the MBP, respectively.

\section{Discussion}

Protein Binding. A comparison of the binding of the MBP and two BNPS-skatole-cleaved fragments of the MBP to DMPG bilayers is given in Figure 1. All three proteins were found to bind strongly to DMPG bilayers consistent with earlier reports on MBP binding to acidic lipids (Palmer \& Dawson, 1969; Sankaram et al., 1989). Comparison of the binding stoichiometries and strengths of binding gives further information on the mode of interaction of the MBP with negatively charged lipids.

The lipid/protein stoichiometry of the complexes at saturation protein binding is $36 \mathrm{~mol}$ of DMPG/18.4-kDa MBP, $24 \mathrm{~mol}$ of DMPG/12.4-kDa fragment $F 1$, and $11 \mathrm{~mol}$ of DMPG $/ 5.8-\mathrm{kDa}$ fragment $\mathrm{F} 2$. These values correspond reasonably closely to the total number of positive charges on the various protein fragments: 31 for MBP, 20 for fragment $F 1$, and 11 for fragment F2. For MBP and fragment F1, the positive residues on the protein do not quite compensate the total number of negative charges contributed by the lipid, whereas for the fragment F2, which has the highest positive charge density per unit protein mass, an equality of charge is achieved. Experiments on $\mathrm{Mn}^{2+}$ binding have revealed that $26 \pm 5,17 \pm 3$, and $11 \pm 1.5$ lipid molecules per protein molecule are protected from binding on association of MBP, F1, and F2, respectively (Boggs et al., 1981). These values agree reasonably well with the total positive charges on the different fragments and further support the above conclusions regarding the charge stoichiometry at saturation binding.

The binding stoichiometries for the different protein fragments are practically equivalent on a mass basis. This is seen from the fact that the stoichiometries of the F1 and F2 fragments sum to that of the whole protein. It was previously calculated that the $36: 1 \mathrm{~mol} / \mathrm{mol}$ saturation binding of the whole MBP corresponded to practically complete coverage of the surface of the bilayer (Sankaram et al., 1989). This result was based on the dimensions of the MBP in solution determined by Epand et al. (1974). The equivalence of the stoichiometries for the two fragments on a mass basis $(35 \mathrm{~mol}$ of DMPG corrected to a protein molecular weight of $18.4 \mathrm{~K}$ ) with that of the whole MBP (36 mol of DMPG/18.4-kDa protein) suggests, therefore, that the fragments also completely cover the bilayer surface at saturation binding. The question thus arises as to whether the saturation binding at the lipid surface is sterically limited. The results of the salt dependence (Figure 5) indicate that this is not the case. Immediately that the electrostatic interaction is partially screened by ions, the binding of the protein decreases; there is no threshold which must first be reached before the binding decreases. Instead, a certain minimum degree of electrostatic attraction seems to be required for binding: at saturation binding, the net surface charge density of the complexes still remains negative and has values of -0.44 e, -0.46 e, and -0.36 e per lipid for the MBP, F1 fragment, and F2 fragment, respectively. These values are rather similar, at least for $\mathrm{MBP}$ and $\mathrm{F} 1$, and support the idea of a minimum threshold electrostatic interaction being required.

The results of Figure 1 show that the binding of all the fragments is very tight at low protein to lipid ratios (the linear region of the binding curves). All the protein which is added is bound, indicating that the binding constant lies well below the concentration range at which the ESR experiments and the binding assays were performed. As the binding approaches saturation, there are marked deviations from linearity, which indicate that the strength of binding is in the order MBP > F1 $>$ F2 (see Figure 1). This result is to be expected on electrostatic grounds. The effective binding constant, $K$, is enhanced relative to the intrinsic binding constant, $K_{0}$, by the electrostatic attraction of the free protein to the charged lipid surface [see, e.g., Cevc and Marsh (1987)]:

$$
K=K_{0} \exp (-Z e \Phi / k T)
$$

where $\Phi$ is the (negative) surface potential of the bilayer, $Z e$ is the net (positive) charge on the protein fragment, and other symbols have their usual meaning. The values of the net charge on the fragments are $Z=+20,+13$, and +7 for the $\mathrm{MBP}$, the F1, and the F2 fragments, respectively. This is in the same order as the strength of binding.

The binding of the protein reduces the bilayer surface potential due to its compensating net positive charge. Therefore, as more protein is bound, the binding becomes weaker according to eq 3 . As the binding approaches saturation, the binding constants can be estimated to be approximately 1.5 , 0.2 , and $0.1 \mathrm{mM}^{-1}$ for the MBP, F1, and F2 fragments, respectively. (These values are low because the surface charge has already been reduced by the protein binding, as is seen from the deviation of the calculated binding curves from the data points in the linear region of Figure 1.) The corresponding values of $\ln K$ are in the ratio (MBP:F1:F2) 1:0.7:0.6, as compared with the values of $Z$ which are in the ratio 1:0.65:0.35. These ratios would be expected to be the same only if the values of $K_{0}$ for the different fragments are the same and if the measurements are made at the same values of the net surface potential (cf. eq 3). Fragment F1, but not fragment F2, seems to conform to these conditions.

Salt Dependence of Protein Binding. Further information about the electrostatic contributions to the strength of binding can be obtained from the studies of the ionic strength dependence. The results of Figure 5 indicate that the binding of the F2 fragment is most sensitive to ionic strength, with the order of sensitivity being $\mathrm{F} 2>\mathrm{Fl}>\mathrm{MBP}$. These results can also be understood, at least qualitatively, from eq 3 , if it is assumed that a certain minimum value of the exponential electrostatic enhancement factor is required for binding to occur. Since the size of this term is determined by the value of the net charge, $Z$, on the protein fragment, this minimum value will be reached at lower values of ionic strength for the fragments with lower values of $Z$. In the low-potential approximation of Gouy-Chapman diffuse double-layer theory, which should be approximately valid at saturation binding, the surface potential is given by [see, e.g., Cevc and Marsh $(1987)]^{2}$

$$
\Phi=(\sigma / e)\left(k T / 2000 \epsilon \epsilon_{0} N_{\mathrm{A}}\right)^{1 / 2} T^{-1 / 2}
$$

where $\sigma$ is the bilayer surface charge density and $I$ is the ionic strength of the electrolyte solution, and other symbols have their usual meaning. If the product $Z \Phi$ in eq 3 is to be kept constant, the ionic strength at which binding is completely abolished therefore should be proportional to $Z^{2}$, i.e., in the ratio 1:0.42:0.12 for MBP, F1, and F2, respectively. This is qualitatively in agreement with the data for the salt concentration required for full displacement given under Results. The limiting ratios of the ionic strength required for complete

\footnotetext{
${ }^{2}$ Gouy-Chapman theory assumes a uniform planar surface charge distribution. This is a simplistic approach to the discrete charge effects which will be present in a protein-containing surface. It is used here only to approximate the dependence on net surface charge.
} 
displacement of the protein are 1:0.5:0.3 for the MBP, F1, and $F 2$, respectively, again indicating a deviation in the case of fragment $F 2$.

Thus, cleavage of the MBP at the tryptophan residue does not affect the stoichiometry of binding at low ionic strength. However, it does affect the strength of the electrostatic contribution to the binding, in a manner which is reflected by the ionic strength dependence in Figure 5.

Protein Penetration. Well-defined two-component ESR spectra indicative of protein penetration into lipid bilayers were observed only with the native protein and the N-terminal fragment, $\mathrm{Fl}$. The stoichiometries of the motionally restricted lipid are $18: 1 \mathrm{~mol} / \mathrm{mol}$ for the MBP and 9:1 $\mathrm{mol} / \mathrm{mol}$ for the F1 fragment. This indicates that cleavage at the Trp residue leads to a disruption of the hydrophobic interaction when compared to the native protein, since the fragment F2 contributes little to the observed motionally restricted component. It therefore seems likely that the membrane-penetrating section of the MBP is not located solely within fragment Fl but also involves residues in the region of the point of cleavage.

The interpretation that the tryptophan residue is located in folded regions of the MBP which interact hydrophobically with the bilayer membrane is in agreement with earlier reports. A blue shift observed for the emission maximum of the tryptophan in the presence of an anionic detergent, sodium dodecyl sulfate, has indicated that this residue is in the hydrocarbon region of the detergent (Jones \& Rumsby, 1975). Similar spectral shifts have also been observed when the phosphorescence of the MBP was monitored in the presence and absence of phosphatidylserine vesicles (Vadas et al., 1981).

It is of interest to compare the relative results on the different fragments with the amino acid sequence of the protein. Plots of the mean hydrophobicity, and of the hydrophobic moments based on either an $\alpha$-helical or a $\beta$-sheet structure, are given in Figure 6 . The MBP is a highly polar protein, and no stretches longer than 14 residues are found which have a net positive hydrophobicity when measured on the consensus scale of Eisenberg et al. (1982). These results are essentially in accordance with those of Weise (1985) which were obtained with a different hydrophobicity scale. In Figure 6, the average hydrophobicities and hydrophobic moments are taken over a window of nine adjacent residues. Two regions with net positive hydrophobicity are obtained, namely, residues $36-46$ and 85-96, which are both located in the F1 fragment. The principal hydrophobic segment is that around residue 90 , which contains the only appreciable hydrophobic cluster, $\mathrm{Val}^{85} \mathrm{ValHis} \mathrm{PhePhe}{ }^{90}$, and corresponds to a very low value of the hydrophobic moment for both $\alpha$-helical and $\beta$-sheet structures. Thus, the stretch $85-96$ is not amphiphilic and, therefore, is a reasonable candidate for a membrane-penetrating section of the protein. The stretch of residues 36-46 has a somewhat higher hydrophobic moment. Two other shorter stretches of the sequence have a net positive hydrophobicity. These are residues 14-21 in the F1 fragment (when calculated with an eight-residue window rather than the nine-residue window shown in Figure 6) and residues 148-154 in the F2 fragment. Both of these stretches also have a low value of the hydrophobic moment. It is noteworthy that the region of protein cleavage has a relatively low hydrophobicity, although it has a moderately high value of the hydrophobic moment, which is one characteristic of an amphiphilic structure.

The fact that MBP and fragments can be wholly displaced from the membrane by salt indicates that the hydrophobic interactions themselves do not make a major contribution to

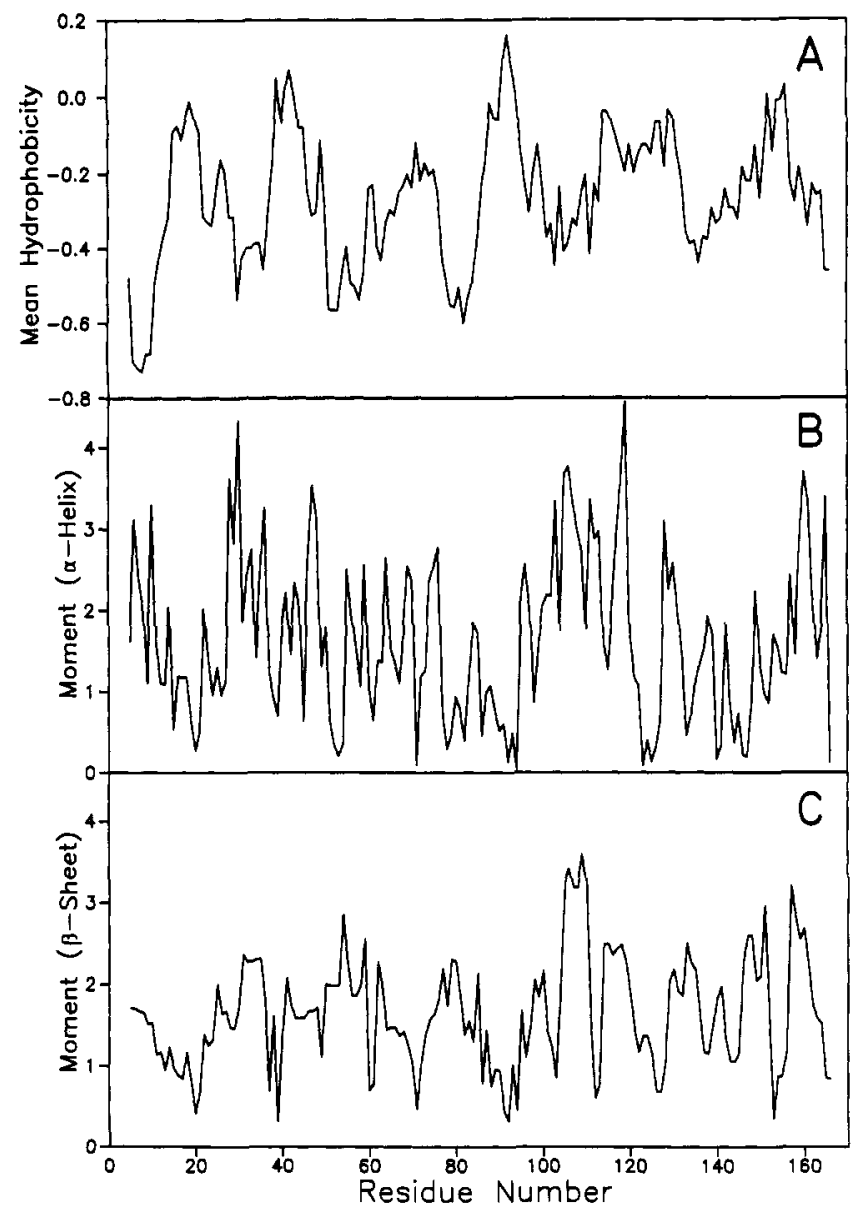

FIGURE 6: Mean hydrophobicity (A) and hydrophobic moment calculated by assuming either an $\alpha$-helical (B) or a $\beta$-sheet (C) conformation for the amino acid sequence of myelin basic protein (Eylar et al., 1971). Values are calculated according to Eisenberg et al. (1982) and are averaged over a span of nine residues centered on each sequence position.

the binding energy. However, it is possible that penetration of part of the protein into the membrane does allow an optimum electrostatic interaction between lipid headgroups and protein side chains.

Lipid Chain Mobility. The decrease in mobility of the 5-PGSL spin-label at saturation protein binding is greater for the intact protein than for the fragments, in the order MBP $>\mathrm{F} 1>\mathrm{F} 2$. Thus, although the saturation binding is equivalent in terms of protein mass for all the proteins, the size of the individual fragments affects the degree to which the lipid packing density, and hence acyl chain mobility, is perturbed. This probably follows from some sort of loose cross-linking of the lipid headgroups by interaction with the charged protein side chains. The extent of linking would then depend on the size of the protein and its number of positively charged residues.

The mobility of the lipid chains interacting hydrophobically with the protein is modulated in a rather different way. The spectrum of the motionally restricted lipid component is very similar for both the MBP and the F1 fragment (cf. Figure 4). The two proteins differ, rather, in the number of lipids whose chains interact directly with the protein. For the F2 fragment, on the other hand, the hydrophobically interacting lipid component either is below the minimum limit for detection or has a mobility which is difficult to resolve from that of the remaining lipid pool.

Conclusions. The different ionic strength dependences of binding for the various fragments demonstrate that the in- 
teraction of the MBP with negatively charged lipid bilayers is principally electrostatic in nature, being determined to a large extent by the net charge on the protein or its fragments. At least one of the sites of membrane penetration and hydrophobic interaction with the lipid is situated in the Fl fragment of the protein, and it seems likely that another corresponds to the region around the point of cleavage between the $\mathrm{F} 1$ and $\mathrm{F} 2$ fragments. Although the saturation binding levels are identical for the different fragments, on a mass basis, the larger fragments cause a greater perturbation of the lipid mobility. The intact protein probably contributes more to the stability of the myelin structure than the sum of its component parts.

\section{ACKNOWLEDGMENTS}

We thank S. Chatterjee for her skilled technical assistance in the preparation of the protein.

Registry No. DMPG, 61361-72-6; tryptophan, 73-22-3.

\section{REFERENCES}

Boggs, J. M., \& Moscarello, M. A. (1978a) J. Membr. Biol. $39,75-96$.

Boggs, J. M., \& Moscarello, M. A. (1978b) Biochim. Biophys. Acta 515, 1-21.

Boggs, J. M., Wood, D. D., \& Moscarello, M. A. (1981) Biochemistry 20, 1065-1073.

Boggs, J. M., Moscarello, M. A., \& Papahadjopoulos, D. (1982) in Lipid-Protein Interactions (Jost, P. C., \& Griffith, O. H., Eds.) Vol. 2, pp 1-51, Wiley, New York.

Cevc, G., \& Marsh, D. (1987) Phospholipid Bilayers. Physical Principles and Models, 442 pp, Wiley-Interscience, New York.

Chao, I. P., \& Einstein, E. R. (1970) J. Neurochem. 17, $1121-1132$.

Chou, C.-H. J., Chou, F. C.-H., Kowalski, T. J., Shapira, R., \& Kibler, R. F. (1977) J. Neurochem. 28, 115-119.

Comfurius, P., \& Zwaal, R. F. A. (1977) Biochim. Biophys. Acta 488, 36-42.

Deibler, G. E., Martenson, R. E., \& Kies, M. W. (1972) Prog. Biophys. Biochem. 2, 139-165.

Eibl, H., \& Lands, W. E. M. (1969) Anal. Biochem. 30, 51-57.

Eisenberg, D., Weiss, R. M., Terwilliger, T. C., \& Wilcox, W. (1982) Faraday Symp. Chem. Soc. 17, 109-120.

Epand, R. M., Moscarello, M. A., Zierenberg, B., \& Vail, W. J. (1974) Biochemistry 13, 1264-1267.

Eylar, E. H., \& Thompson, M. (1969) Arch. Biochem. Biophys. 129, 469-479.
Eylar, E. H., Brostoff, S., Hashim, G., Caccam, J., \& Burnet, P. (1971) J. Biol. Chem. 246, 5770-5784.

Görrissen, H., Marsh, D., Rietveld, A., \& de Kruijff, B. (1986) Biochemistry 25, 2904-2910.

Horvāth, L. I., Brophy, P. J., \& Marsh, D. (1988) Biochemistry 27, 46-52.

Jones, A. J. S., \& Rumsby, M. G. (1975) J. Neurochem. 25, 565-572.

Jones, A. J. S., \& Rumsby, M. G. (1977) Biochem. J. 167, 583-591.

Keniry, M. A., \& Smith, R. (1979) Biochim. Biophys. Acta 578, 381-391.

Laemmli, U. K. (1970) Nature (London) 227, 680-685.

Lange, A., Marsh, D., Wassmer, K.-H., Meier, P., \& Kothe, G. (1985) Biochemistry 24, 4383-4392.

Liebes, L. F., Zand, R., \& Phillips, W. D. (1975) Biochim. Biophys. Acta 405, 27-39.

London, Y., Demel, R. A., Geurts van Kessel, W. S. M., Vossenberg, F. G. A., \& van Deenen, L. L. M. (1973) Biochim. Biophys. Acta 311, 520-530.

Lowry, O. H., Rosebrough, N. J., Farr, A. C., \& Randall, R. J. (1951) J. Biol. Chem. 193, 265-275.

Marsh, D. (1981) in Membrane Spectroscopy (Grell, E., Ed.) pp 51-142, Springer-Verlag, Berlin.

Marsh, D. (1982) Tech. Life Sci.: Biochem. B4/II, B426/ $1-B 426 / 44$.

Marsh, D. (1985) in Progress in Protein-Lipid Interactions (Watts, A., \& de Pont, J. J. H. H. M., Eds.) pp 143-172, Elsevier, Amsterdam.

Marsh, D. (1986) in Supramolecular Structure \& Function (Pifat-Mrzlijak, G., Ed.) pp 48-62, Springer-Verlag, Berlin.

Marsh, D., \& Watts, A. (1982) in Lipid-Protein Interactions (Jost, P. C., \& Griffith, O. H., Eds.) Vol. 2, pp 53-126, Wiley-Interscience, New York.

Martenson, R. E., Deibler, G. E., Kramer, A. J., \& Levine, S. (1975) J. Neurochem. 24, 173-182.

Palmer, F. B., \& Dawson, R. M. C. (1969) Biochem. J. 111, 629-636.

Sankaram, M. B., Brophy, P. J., \& Marsh, D. (1989) Biochemistry (preceding paper in this issue).

Stone, A. L., Park, J. Y., \& Martenson, R. E. (1985) Biochemistry 24, 6666-6673.

Surewicz, W. K., Moscarello, M. A., \& Mantsch, H. H. (1987) Biochemistry 26, 3881-3886.

Vadas, E. B., Melançon, D., Braun, P. E., \& Galley, W. C. (1981) Biochemistry 20, 3110-3116.

Weise, M. J. (1985) J. Neurochem. 44, 163-170.

Young, P. R., Vaconte, D. A., \& Snyder, W. R. (1982) J. Am. Chem. Soc. 104, 7287-7291. 